\title{
A Randomized Comparison of Circular versus Single Point-By-Point Pulmonary Vein Isolation
}

\author{
Nora Al-Jefairi ${ }^{1}$, Ruairidh Martin ${ }^{1}$, Antonio Frontera ${ }^{1,2}$,Arnaud Denis ${ }^{1}$, Nicolas Derval ${ }^{1}$, Frederic \\ Sacher $^{1,2}$, Saagar Mahida ${ }^{1}$, Stephan Zellerhoff ${ }^{1}$, Xavier Pillois ${ }^{1}$, Meleze Hocini ${ }^{1,2}$, Michel Haïssaguerre ${ }^{1,2}$ \\ and Pierre Jais ${ }^{1,2}$ \\ ${ }^{1}$ Department of Cardiac Pacing and Electrophysiology, Hospital Cardiologique du Haut Leveque, CHU de Bordeaux, Pessac, France
}

${ }^{2}$ IHU LIRYC, University of Bordeaux - Inserm, Pessac, France Received: 01 November, 2017; Accepted: 20 December, 2017; Published: 28 December, 2017
*Corresponding author: Nora Al-Jefairi, Department of Cardiac Pacing and Electrophysiology, Hospital Cardiologique Haut Leveque, Avenue de
Magellan, Pessac, France, Tel: +33 5576564 71; Fax: +33 5765 6509; Email: n.aljefairi@hotmail.com

\begin{abstract}
Introduction: Point-by-point standard ablation using single-tip (conventional) catheter is the main current technique for pulmonary vein isolation (PVI) but it can be challenging. A circular, multielectrode, mapping and ablation catheter (nMARQ) may provide faster PVI.

Methods: We designed a randomized comparison between circular versus conventional PVI with the primary end point of reconnection: acutely, in the index procedure, and at 3 months, at the time of a systematic repeat procedure.

Results: Eighty-one patients randomized to circular (nMARQ n $=38$ ) versus conventional (Thermocool Navistar $n=43$ ) underwent PVI for paroxysmal atrial fibrillation. Both groups had similar characteristics: nMARQ age $61 \pm 11$ years ( $84 \%$ male) and TC age $60 \pm 9$ years (68\% male). NMARQ procedures were shorter (RF and procedure duration), with fewer RIPV reconnections, both acutely and at 3 months (index: $1(3 \%)$ versus 7 (16\%), p = 0.044, redo: $21(18 \%)$ versus $36(28 \%), p=0.05)$. Patients ablated with the circular catheter also had shorter repeat procedures suggesting more discrete PV reconnections (nMRAQ: $142 \pm 56$ versus TC: $186 \pm 76$ minutes, $p$ $=0.001$ ). At 12 months follow up, $77 \%$ of patients in nMARQ group and $79 \%$ in TC group were in SR ( $\mathrm{p}=0.852)$. No major procedural complications were observed.
\end{abstract}

Conclusion: This randomized study shows that nMARQ catheter is 3 times faster in isolating the PVs, with shorter RF and procedure time. It is also associated with fewer PV reconnections at 3 months. NMARQ appeared to be safe with no major procedural complications.

Keywords: Paroxysmal atrial fibrillation; Pulmonary vein isolation; Circular (nMARQ) catheter ablation; Single point-by-point (Thermocool catheter ablation); Reconnection rate

\section{Introduction}

Electrical Pulmonary Vein Isolation (PVI) by catheter ablation, to eliminate trigger activity or to alter arrhythmogenic substrate, is the mainstay of treatment for patients with symptomatic and drug refractory Paroxysmal Atrial Fibrillation (PAF) [1].

However, Atrial Fibrillation (AF) recurrence after PVI is common $(20-55 \%)$ and generates significant extra costs, including repeat hospital admissions and repeat procedures. PV electrical reconnection is considered the dominant mechanism of recurrence that limits long-term success and remains a current issue despite advances in catheter design, ablation techniques and AF treatment strategies [2-7]. Other potential mechanisms of late recurrence have been described, due to electrical and structural atrial remodelling as a result of aging, heart failure, inflammation, diabetes and obesity $[3,8,9]$.

Recent catheter technologies such as catheter localization, catheter stability and contact force monitoring might be associated with better and more durable Radio Frequency (RF) lesions at first attempt.

Point-by-point standard ablation using single-tip (conventional) catheter is the main current technique for PVI but it can be challenging, complex and time-consuming. A circular, multi-electrode, mapping and ablation catheter (nMARQ) was developed, which provides fast and effective PVI with comparable success and safety [10]. To the best of our knowledge, the circular ablation catheter has not been compared to the conventional point-by-point approach in a randomized study of PVI. Moreover, the acute and late reconnection rate using circular catheter versus point-by-point ablation remains unknown. We designed such a randomized comparison with the primary end point of reconnection rate: acutely, in an index procedure, and at 3 months, in a repeat procedure.

\section{Methods}

\section{Study Population}

Patients $\geq 18$ years, suffering from symptomatic, drug refractory PAF (self-terminating episodes of $<7$ days), as defined by ACC/AHA/ESC (American College of Cardiology/ American Heart Association/ European Society of Cardiology) guidelines were eligible to participate in the study. Exclusion criteria were: Left Atrial (LA) thrombus, severe mitral valvulopathy, class III/IV NYHA (New York Heart Association) heart failure, oral anticoagulation contraindication, redo-ablation, untreated 
psychiatric disorders and pregnancy. Patients were provided with written informed consent and were affiliated to or recipients of a social welfare regimen [5]. The study was approved by the Institutional Clinical Research and Ethics Committee.

\section{Index Ablation Procedure}

All patients were anticoagulated for at least 1 month before the procedure. Oral vitamin $\mathrm{K}$ anticoagulation was uninterrupted for the procedure. LA thrombus was excluded either by Transoesophageal Echocardiography (TEE) or Cardiac Computerized Tomography (CCT) $<5$ days prior to the procedure. Antiarrhythmic Drugs (AADs), excepting amiodarone, were discontinued for $\geq 5$ half-lives before the procedure.

Conscious sedation was achieved with intravenous midazolam $(0.2 \mathrm{mg} / \mathrm{kg})$, morphine sulphate $(0.1 \mathrm{mg} / \mathrm{kg}$ to $0.2 \mathrm{mg} / \mathrm{kg})$ and, if necessary, sufentanil (by $5 \mu \mathrm{g}$ up to $20 \mu \mathrm{g}$ ) with continuous noninvasive vital sign monitoring. Surface Electrocardiograms (ECG) and bipolar endocardial ECG were continuously displayed and recorded at a sweep speed of $100 \mathrm{~mm} / \mathrm{s}$ and filtered from 0.05 to $100 \mathrm{~Hz}$ and 30 to $250 \mathrm{~Hz}$, respectively (LabsystemProTM EP recording system, Boston Scientific BSCI, MA, USA).

Using bupivacaine hydrochloride local anaesthesia (100 $\mathrm{mg} / 20 \mathrm{ml}$ subcutaneous SC), three femoral vein sheaths were inserted $(8 \mathrm{~F}, 7 \mathrm{~F}$ and $6 \mathrm{~F})$ for vascular access. A steerable quadripolar catheter (Xtrem Sorin, France or Dynamic, Boston scientific, Marlborough, MA, USA) was positioned inside the Coronary Sinus (CS) through the 6F sheath. A single Transeptal Puncture (TSP) was obtained using a $98 \mathrm{~cm}$ BRK needle (St. Jude Medical) inserted through a long sheath (Agilis, St. Jude Medical) for patients randomized to nMARQ. For those randomized to point-by-point ablation, the transeptal puncture was carried out using a $78 \mathrm{~cm}$ BRK needle and a SL0 long sheath (St. Jude Medical). Access to the LA was confirmed by pressure monitoring and contrast injection (Lobitridol $300 \mathrm{mg} \mathrm{l} / \mathrm{mL}$ ) under fluoroscopy. The long sheath was continuously perfused with heparinized saline $(2500 \mathrm{U}$ for $500 \mathrm{ml})$ at $200 \mathrm{~mL} / \mathrm{h}$ to reduce the risk of thrombus formation. Immediately after TSP and guide wire insertion, a bolus of intravenous heparin $(0.5-0.8 \mathrm{mg} / \mathrm{kg}$ ) was injected. Heparin was monitored throughout the procedure every 20 to 30 minutes by Activated Clotting Time (ACT) with a target of 300-400 seconds.

According to treatment group, either a circular (nMARQ®, Biosense Webster) or a conventional (NAVISTAR $\AA$ THERMOCOOL®, Biosense Webster) catheter was used for mapping and ablation. When the nMARQ catheter was used, it was inserted through the long sheath.

In the conventional group, a mapping circular catheter (Lasso ${ }^{\circledR}$, Biosense Webster) catheter was introduced through the long sheath (described below).

\section{Mapping and Ablation}

LA and PV anatomy were then reconstructed using Fast Anatomical Mapping (FAM) with nMARQ or Lasso catheter (according to randomization) connected to the Carto $\AA 3$ Navigation System (Biosense Webster, Diamond Bar).

The nMARQ catheter has 10 open irrigated electrodes used for both mapping and ablation (electrode length $3.5 \mathrm{~mm}$, spacing 4 $\mathrm{mm}$, maximum catheter diameter $8.4 \mathrm{~F}$ ) arranged on an adaptable circle (diameter $20-35 \mathrm{~mm}$ ), to allow optimal positioning in the PV antrum (Figure 1 and 2) [10]. The catheter was irrigated at $60 \mathrm{~mL} / \mathrm{min} 0.9$ saline during RF delivery (Cool Flow, Biosense Webster). The generator (nMARQ Generator, Biosense Webster) delivers RF energy through the 10 separated electrodes in unior bipolar mode. Maximum power used was 25 Watt (W) (20 $\mathrm{W}$ for posterior wall to reduce the risk of oesophageal injury), temperature $45^{\circ} \mathrm{C}$, maximum duration time 60 seconds in unipolar mode. Catheter position, placement and contact was monitored and optimized before any RF application. Contact was assessed visually with fluoroscopy and also by impedance-based technology provided by Carto ${ }^{\circledR} 3$ (Tissue Connect, Biosense Webster). RF parameters were continuously monitored during ablation and intermittent fluoroscopy was obtained to assess diaphragm movement, in order to prevent right phrenic nerve injury during right PV ablation. For confirmation of PV isolation, the circular catheter was moved slightly more distally before and after ablation (using the smallest diameter) to verify far-field signals and assess for entrance block. Pacing for phrenic nerve capture and oesophageal temperature monitoring were not routinely used.

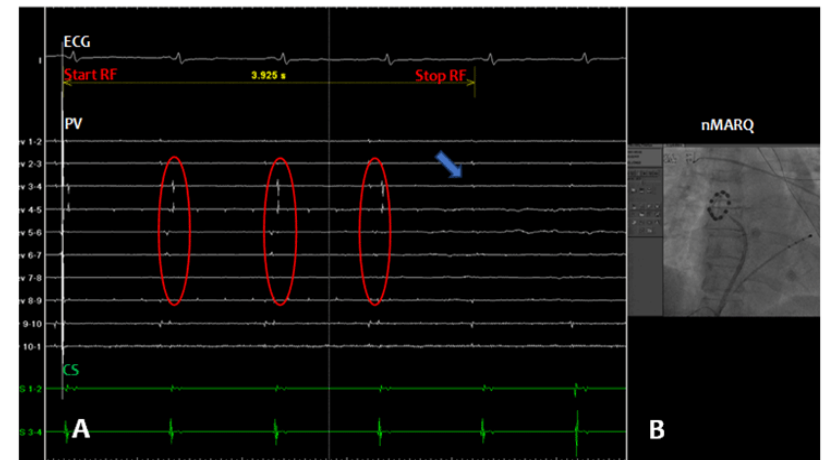

Figure 1: (A) disconnection of PV potentials (red circles) after 4 seconds in SR by nMARQ ablation catheter in the RSPV (shown by blue arrow); (B) AP x-ray view showing nMARQ catheter position in RSPV and quadripolar catheter ablation position in the CS

ECG: Surface Electrocardiogram; PV: Pulmonary Vein; RSPV: Right Superior Pulmonary Vein; SR: Sinus Rhythm; CS: Coronary Sinus; AP: AnteroPosterior

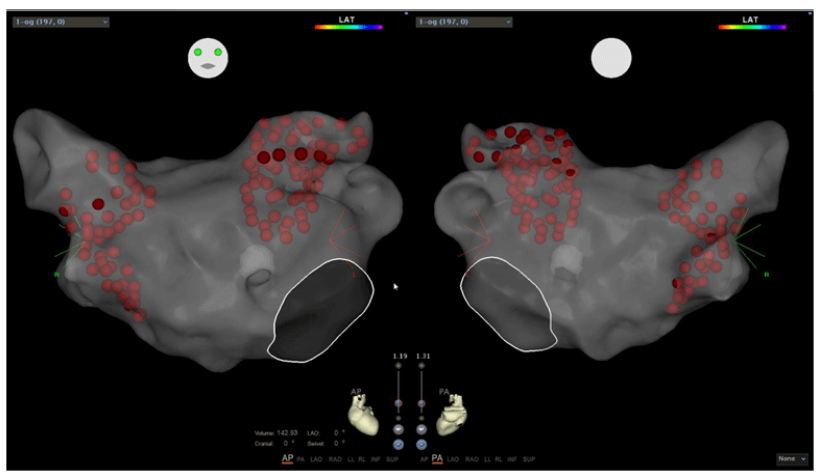

Figure 2: Left atrial 3D geometrical mapping in AP and PA view displaying the location of ablation lesions in PV using nMARQ catheter in index procedure

AP: Antero-Posterior; PA: Postero-Anterior 
Navistar Thermocool (TC) is an open loop, irrigated deflectable, single electrode mapping and ablation catheter $(3.5$ $\mathrm{mm}$ tip and 3 ring electrodes, catheter diameter $7.5 \mathrm{~F}$ ). Catheter irrigation was delivered by a pump (baseline $2 \mathrm{~mL} / \mathrm{min}, 8-15$ $\mathrm{mL} / \mathrm{min}$ during energy delivery, $0.9 \%$ saline via Cool Flow ${ }^{\circledR}$, Biosense Webster). RF parameters were: power $30 \mathrm{~W}(25 \mathrm{~W}$ on the posterior wall), max temperature $52^{\circ} \mathrm{C}, \mathrm{RF}$ duration time 30-60 seconds. With the ablation catheter, we used a 10-pole circular catheter, (Lasso) for PV mapping. PVI was achieved by creating a wide antral line around ipsilateral PVs combining point-by-point and dragging ablation techniques depending on operator preference. The ablation of the inter-vein area was optional (Figure 3). Targeting the earliest PV potential activation approximately 5 to $10 \mathrm{~mm}$ from PV ostium was sometimes performed to target a residual gap and complete the isolation.

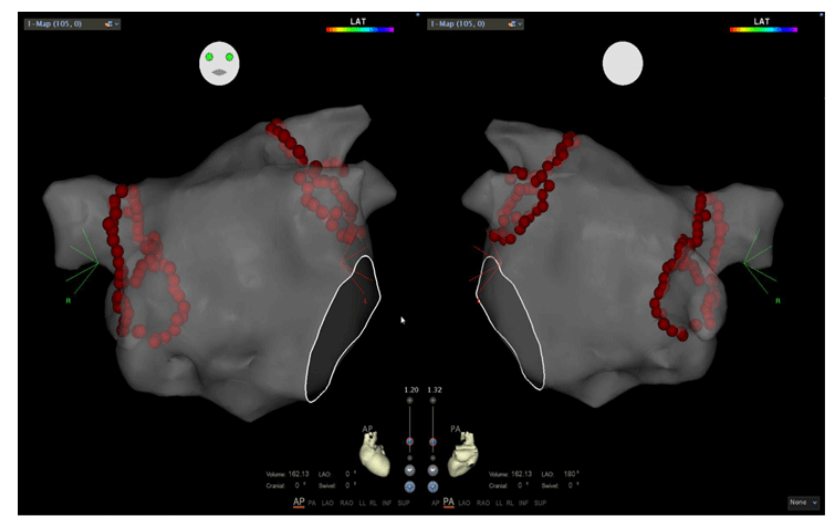

Figure 3: Left atrial 3D geometrical mapping in AP and PA view displaying the location of ablation lesions in PV using Thermocool catheter in index procedure

AP: Anteroposterior; PA: Posteroanterior

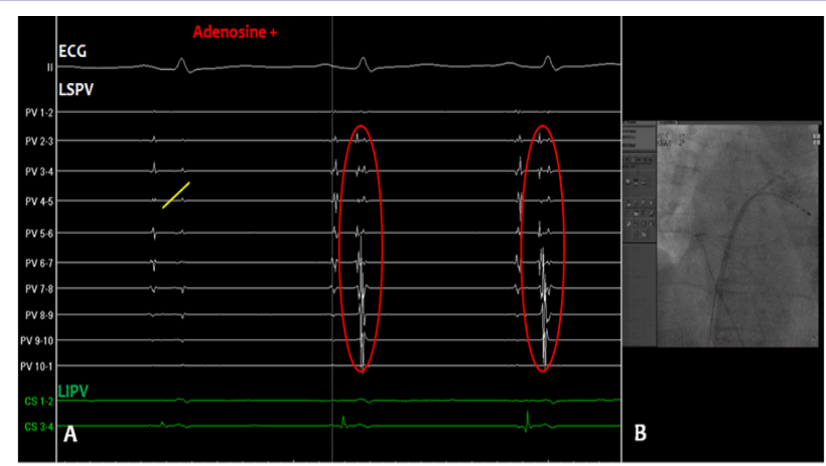

Figure 4: (A) PV potential (second signal component in red circles, first signal component is atrial far-fields) recovery after 30 minutes of adenosine injection. PV disconnection is shown by yellow line; (B) AP x-ray view showing Lasso mapping catheter position in LSPV and quadripolar catheter ablation position in the LIPV to assess reconnection in LPV after adenosine injection.

ECG: Surface Electrocardiogram; PV: Pulmonary Vein; LSPV: Left Superior Pulmonary Vein; LIPV: Left Inferior Pulmonary Vein; SR: Sinus Rhythm; AP: Anteroposterior

RF duration, in minutes, was evaluated and compared in 3 ways: each vein separately: Right Superior PV (RSPV), Right Inferior PV (RIPV), Left Superior PV (LSPV) and Left Inferior PV
(LIPV); ipsilateral pulmonary vein pairs, RPV and LPV; and all PVs together. PV electrical disconnection was confirmed by lasso catheter and pacing for entrance block, exit block, and reconfirmed systematically after 30 minutes with adenosine injection (in absence of contraindication) (Figure 4). Reconnected veins after adenosine injection were re-ablated targeting the earliest PV potential region. Disconnection was again demonstrated by entrance and exit block, and adenosine injection.

Cavotricuspid Isthmus (CTI) ablation was performed for patients with documented typical atrial flutter in both groups. LA defragmentation \pm Direct Current cardioversion (DC) shock/ AADs were allowed if AF persisted after PVI. The end point of LA defragmentation was to reduce signal complexity and to prolong the local cycle length, to finally achieve AF termination to SR or atrial tachycardia.

\section{Redo Procedure at 3 Months}

Patients were systematically offered a second procedure irrespective of symptoms or recurrence to evaluate PV for electrical reconnection using conventional strategy. No nMARQ catheters were used in repeat procedures.

The procedure was performed as for the index procedure with conventional catheters. LA and PV 3D anatomy were reconstructed and high-density anatomical mapping was acquired with Carto 3 system using TC and/or Lasso NAV. Each $\mathrm{PV}$ antral region was carefully evaluated for isolation or electrical reconnections/gaps in PV line using Lasso mapping catheter. PVs were divided into 4 segments (superior, anterior, inferior and posterior) and each segment with reconnection was targeted with RF ablation (TC) until complete PVI was achieved (Figure 5). PVI was confirmed locally by the Lasso catheter, with pacing for entrance and exit block and adenosine as explained in index procedure.

The CTI line if present was rechecked for bidirectional block and re-ablated again if necessary. Additional LA defragmentation

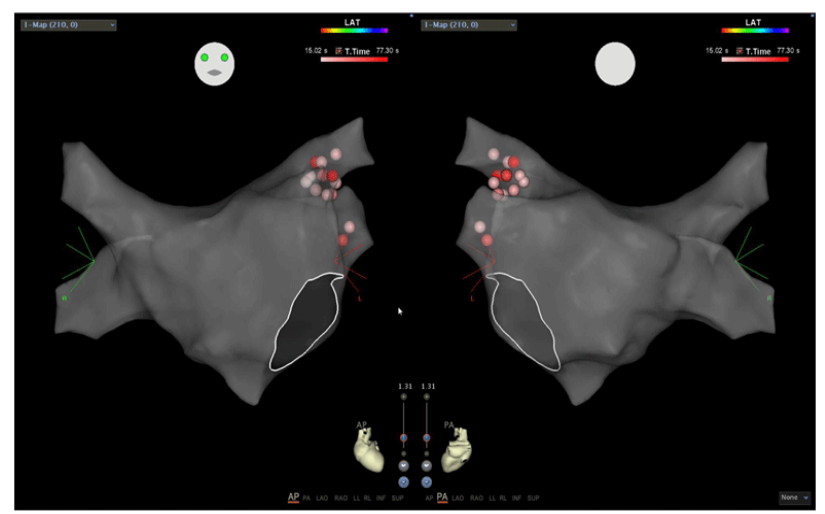

Figure 5: Left atrial 3D geometrical mapping in AP and PA view displaying the location of ablation lesions in PV reconnected segments using Thermocool catheter in redo procedure.

AP: Anteroposterior; PA: Posteroanterior

\section{Clinical Follow Up, Recurrence and Complications}

Following ablation, patients continued oral anticoagulation and were advised to continue AADs for 1 to 3 months to control 
transient tachyarrhythmia episodes during the blanking period Out-patient clinical follow up (FU) evaluation for symptoms and 24 h Holter monitoring was performed systematically at 3, 6 and 12 months after the procedure. At 1 month, patients were evaluated for PV stenosis by contrast-enhanced Magnetic Resonance Imaging (MRI). Complications related to the procedures were recorded to respect local regulatory requirements for safety and diligence.

\section{End Points}

The study primary end point was to compare the efficacy of circular versus conventional ablation catheters for PVI in patients with PAF and to assess reconnection rate: acutely, in the index procedure; and at 3 months, at the repeat procedure and to compare PV reconnection between both procedures. Secondary end point was to assess clinical recurrence 3 months, 6 months and 12 months in both groups.

\section{Statistical Analysis}

Continuous variables were expressed as mean $\pm \mathrm{SD}$ Categorical data were expressed as counts and percentages.
Continuous variables were compared using Student $t$ tests or the non-parametric Mann-Whitney U test as appropriate. Categorical variables were compared using Fisher's exact or Pearson's chi-square tests as appropriate. All univariate predictors with p-values $<0.05$ were considered statistically significant. All statistical analyses were performed using SPSS version 21.0 (SPSS, Inc.,) and Prism version 5.00 (GraphPad Software). To test the correlation between PV reconnection sites in index and repeated procedures, the Cohen's kappa correlation coefficient was calculated.

\section{Results}

\section{Study Population}

Eighty-one patients with symptomatic, drug refractory paroxysmal AF were enrolled in the study and referred for PVI. Thirty-eight patients were randomly assigned to PV ablation with the nMARQ catheter and 43 patients to PV ablation with the TC catheter. The majority of patients were at low clinical cardiovascular profile risk (CHA2DS2-VASc $<2$ ), mild structural heart disease and normal or near normal Left Ventricular Ejection Fraction (LVEF) (Table 1).

Table 1: Baseline patient characteristics

\begin{tabular}{|c|c|c|c|}
\hline $\mathbf{N}=\mathbf{8 1}$ & nMARQ (n 38) & Navistar TC (n 43) & $\mathbf{p}$ \\
\hline Male Sex, n (\%) & $32(84)$ & $29(68)$ & 0.081 \\
\hline Age, mean \pm SD (years) & $61 \pm 11$ & $60 \pm 9$ & 0.613 \\
\hline $\mathrm{BMI}^{*}$, mean \pm SD $\left(\mathrm{kg} / \mathrm{m}^{2}\right)$ & $25.5 \pm 3.8$ & $26.8 \pm 3.8$ & 0.118 \\
\hline Hypertension, n (\%) & $8(21)$ & $13(30)$ & 0.347 \\
\hline Diabetes Mellitus, n (\%) & $1(3)$ & $1(2)$ & 0.929 \\
\hline Embolic events, n (\%) & $3(8)$ & $5(12)$ & 0.574 \\
\hline Structural heart disease, n (\%) & & & 0.555 \\
\hline Ischemic heart disease & $4(11)$ & $1(2)$ & 0.126 \\
\hline Dilated or hypertrophic cardiomyopathy & $2(5)$ & $1(2)$ & 0.484 \\
\hline Valvular heart disease & $5(13)$ & $6(14)$ & 0.917 \\
\hline Left ventricular ejection fraction, mean \pm SD (\%) & $63 \pm 6$ & $61 \pm 6$ & 0.231 \\
\hline Left atrial surface area, mean \pm SD $\left(\mathrm{cm}^{2}\right)$ & $18.9 \pm 5.0$ & $19.5 \pm 5.0$ & 0.607 \\
\hline \multicolumn{4}{|l|}{$\mathrm{AF}^{\dagger}$ related parameters } \\
\hline AF history, mean \pm SD (months) & $71 \pm 77$ & $89 \pm 86$ & 0.352 \\
\hline AF max duration episodes, mean \pm SD (hours) & $23.6 \pm 26.9$ & $27.3 \pm 38.7$ & 0.657 \\
\hline $\mathrm{AF}$ at baseline, $\mathrm{n}(\%)$ & $8(21)$ & $8(19)$ & 0.782 \\
\hline$\geq 1$ DC $^{*}$ shock, n (\%) & $4(10)$ & $6(14)$ & 0.743 \\
\hline AADs $^{\dagger}$ used before AF ablation, n (\%) & $36(95)$ & $41(95)$ & 1.0 \\
\hline Amiodarone use before AF ablation, n (\%) & $18(47)$ & $16(37)$ & 0.485 \\
\hline CHA2DS2-VASc, mean \pm SD & $1 \pm 1$ & $1 \pm 1$ & 0.388 \\
\hline
\end{tabular}




\section{Index Ablation Procedure}

At baseline, 8 patients (21\%) in nMARQ group and 8 patients (19\%) in TC group were in AF. Complete PVI was achieved successfully in both groups (100\%), with no cross over.

\section{Radiofrequency Duration for PVI}

RF duration was significantly shorter in nMARQ group, either vein-by-vein or when comparing ipsilateral veins or all 4 veins $(p<0.001)$. Isolation of the LIPV required a shorter RF duration compared to the other PVs in both groups (Table 2).

\begin{tabular}{|c|c|c|c|}
\hline $\min \pm S D$ & nMARQ (n 38) & $\begin{array}{c}\text { Navistar TC } \\
\text { (n 43) }\end{array}$ & $\mathbf{p}$ \\
\hline $\mathrm{RSPV}^{\dagger}$ & $4 \pm 3$ & $11 \pm 8$ & $<0.001$ \\
\hline $\mathrm{RIPV}^{\ddagger}$ & $3 \pm 2$ & $11 \pm 5$ & $<0.001$ \\
\hline $\mathrm{LSPV}^{\S}$ & $3 \pm 2$ & $12 \pm 6$ & $<0.001$ \\
\hline $\mathrm{LIPV}^{* *}$ & $2 \pm 2$ & $8 \pm 5$ & $<0.001$ \\
\hline $\mathrm{RPV}^{\dagger \dagger}$ & $6 \pm 4$ & $20 \pm 9$ & $<0.001$ \\
\hline LPV & $5 \pm 3$ & $18 \pm 8$ & $<0.001$ \\
\hline Total PV & $12 \pm 5$ & $38 \pm 13$ & $<0.001$ \\
\hline \multicolumn{4}{|c|}{ *Radiofrequency } \\
\hline \multicolumn{4}{|c|}{${ }^{\dagger}$ Right superior pulmonary vein } \\
\hline \multicolumn{4}{|c|}{ †Right inferior pulmonary vein } \\
\hline \multicolumn{4}{|c|}{${ }^{\S}$ Left superior pulmonary vein } \\
\hline \multicolumn{4}{|c|}{${ }^{*}$ Left inferior pulmonary vein } \\
\hline \multicolumn{4}{|c|}{${ }^{\dagger+}$ Right pulmonary veins } \\
\hline \multicolumn{4}{|c|}{${ }^{\ddagger}$ Left pulmonary veins } \\
\hline
\end{tabular}

\section{Adenosine and Acute PV Reconnection Rates After Complete PVI}

Thirty minutes after PVI, adenosine challenge was performed in $34(92 \%)$ and $38(88 \%)$ patients in the nMARQ and TC groups respectively.

A lower reconnection rate in RIPV in nMARQ group was observed, compared to TC, $3 \%$ versus $16 \%, p=0.044$ ). RSPV in nMARQ group and LSPV in TC group had a higher reconnection rate when compared to other veins of the same catheter group (Figure 6).

The acute reconnection rate per PV segment was available in 11 (73\%) patients in the nMARQ arm and 17 (74\%) patients in the TC arm as shown in table 1 in supplementary files. The reconnection rate in the anterior segment of RSPV was significantly lower in TC compared to nMARQ group $12 \%$ versus $46 \%$ respectively, $p$ $=0.044)$. The latter had the highest reconnection rate compared to other PV segments in nMARQ group. On the other hand, the inferior segment of RIPV and the anterior segment of LSPV (24\%) had the highest reconnection rate in TC group.

There was no significant difference in the number of acute reconnection per patients between both groups (Figure 7). Seventy percent of patients had no acute reconnection in nMARQ versus $58 \%$ in TC group. In some cases, PV reconnection was limited to one vein ( $22 \%$ with nMARQ versus $30 \%$ with TC). The total number of acutely reconnected PV was higher in the TC group but this was not statistically significant $(p=0.351)$.

\section{Additional Interventions}

Table 3 shows the rate of LA defragmentation, CTI ablation for documented atrial flutter, total non-PV RF duration, DC, and AADs

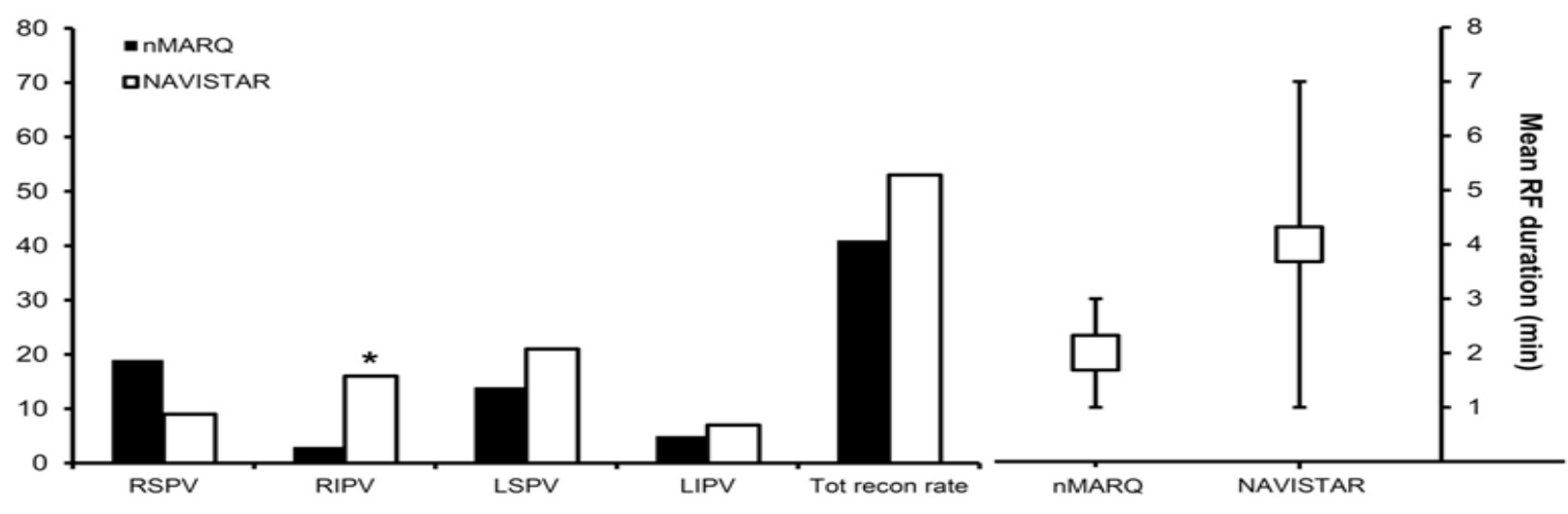

Figure 6: Acute pulmonary vein reconnection per patient in index procedure

(Flecainide) used to restore SR after PVI in both groups shown in table 2 in the supplementary file.

There was no significant statistical difference between both groups in non-PV ablations and DC/AADs. RF duration for nonPV ablation was significantly lower in nMARQ group compared to $\mathrm{TC}(5 \pm 3$ versus $15 \pm 11 \mathrm{~min}, \mathrm{p}=0.014)$, respectively shown in table 3 in the supplementary file.

\section{Procedural Parameters}

Total procedure duration was $111 \pm 35$ versus $202 \pm 52$ minutes ( $p=0.001$, total RF duration was $14 \pm 6$ versus $43 \pm 14$ minutes $(\mathrm{p}=0.001)$ and $\mathrm{X}$-ray exposure was $26 \pm 11$ versus $46 \pm$ 27 minutes $(\mathrm{p}=0.001)$ in $\mathrm{nMARQ}$ versus TC, respectively. 
Supplementary Table 1:Reconnection rate per PV segment in index procedure

\begin{tabular}{|c|c|c|c|c|}
\hline \multicolumn{2}{|c|}{ n (\%) } & nMARQ (n 11) & Navistar TC (n 17) & $\mathbf{p}$ \\
\hline \multirow{4}{*}{ RSPV } & Superior & $0(0)$ & $0(0)$ & - \\
\hline & Anterior & $5(46)$ & $2(12)$ & 0.044 \\
\hline & Inferior & $1(9)$ & $2(12)$ & 0.823 \\
\hline & Posterior & $1(9)$ & $1(6)$ & 0.747 \\
\hline \multirow{4}{*}{ RIPV } & Superior & $0(0)$ & $3(18)$ & 0.14 \\
\hline & Anterior & $0(0)$ & $3(18)$ & 0.14 \\
\hline & Inferior & $0(0)$ & $4(24)$ & 0.082 \\
\hline & Posterior & $1(9)$ & $2(12)$ & 0.823 \\
\hline \multirow{4}{*}{ LSPV } & Superior & $1(9)$ & $2(12)$ & 0.823 \\
\hline & Anterior & $3(27)$ & $4(24)$ & 0.823 \\
\hline & Inferior & $1(9)$ & $2(12)$ & 0.823 \\
\hline & Posterior & $0(0)$ & $0(0)$ & - \\
\hline \multirow{4}{*}{ LIPV } & Superior & $1(9)$ & $1(6)$ & 0.747 \\
\hline & Anterior & $1(9)$ & $1(6)$ & 0.747 \\
\hline & Inferior & $1(9)$ & $1(6)$ & 0.747 \\
\hline & Posterior & $1(9)$ & $1(6)$ & 0.747 \\
\hline
\end{tabular}

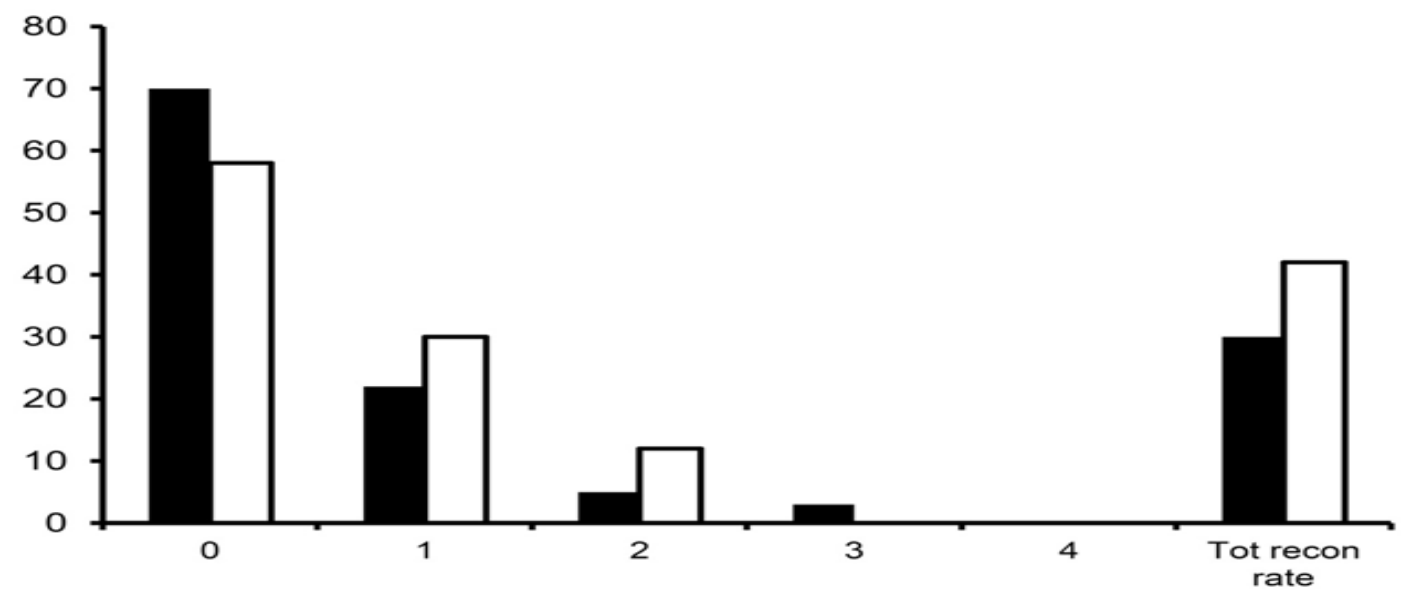

Figure 7: Percentage of acute pulmonary vein reconnection per patient in $4 \mathrm{PV}$ in index procedure

Table 3: Non-PV ablation/cardio version in index procedure

\begin{tabular}{|c|c|c|c|}
\hline & nMARQ (n 37) & Navistar TC (n 43) & $\mathbf{p}$ \\
\hline LA defragmentation, n (\%) & $8(22)$ & $5(12)$ & 0.226 \\
\hline CTI, n (\%) & $10(27)$ & $6(14)$ & 0.145 \\
\hline RF duration, mean $(\min ) \pm S D$ & $5 \pm 3$ & $15 \pm 11$ & 0.014 \\
\hline DC, n (\%) & $2(5)$ & $8(19)$ & 0.075 \\
\hline Flecainide, n (\%) & $2(5)$ & 6 (14) & 0.203 \\
\hline
\end{tabular}




\begin{tabular}{|c|c|c|c|}
\hline \multicolumn{4}{|c|}{ Supplementary Table 2: Patients on AADs at 12 months } \\
\hline n (\%) & nMARQ (n 38 ) & Navistar TC (n 43) & $\mathbf{p}$ \\
\hline On AADS@12 months & $18(45)$ & $20(44)$ & $\mathrm{ns}^{*}$ \\
\hline Flecainide & $4(10)$ & $5(11)$ & ns \\
\hline Amiodarone & $1(3)$ & $1(2)$ & ns \\
\hline Beta blockers & $16(40)$ & $17(38)$ & ns \\
\hline $\mathrm{Ca}^{2+}$ channel blockers & $1(3)$ & $2(4)$ & ns \\
\hline Propafenone & $0(0)$ & $1(2)$ & ns \\
\hline${ }^{*}$ Non significant & & & \\
\hline
\end{tabular}

Supplementary Table 3: Non-PV ablation/cardio version in repeated procedure

\begin{tabular}{|c|c|c|c|}
\hline & nMARQ (n 30) & Navistar TC (n 35) & p \\
\hline LA defragmentation, n (\%) & $5(17)$ & $11(31)$ & 0.168 \\
\hline CTI, $\mathbf{n}$ (\%) & $8(27)$ & $12(34)$ & 0.507 \\
\hline RF duration, mean (min) + SD & $22 \pm 14$ & $20 \pm 16$ & 0.818 \\
\hline DC, $\mathbf{n}$ (\%) & $1(3)$ & $2(6)$ & 0.648 \\
\hline
\end{tabular}

\section{Follow-Up (Redo) Procedure}

Symptomatic recurrence was observed in $17 / 37$ (46\%) and $16 / 43(37 \%)$ in the nMARQ and TC groups, respectively (Table 4). of 81 patients, 62 accepted a repeat procedure at 3 months, 30 patients had PVI previously with nMARQ catheter and 32 patients with TC catheter. At baseline, 2 patients were in AF in nMARQ (7\%) and TC (6\%) group.

\section{PV Reconnection Rate at 3 Months}

The total reconnection rate was significantly lower in the nMARQ group compared to the TC group (15\% versus 20\%, $\mathrm{p}=0.049$ ). The RIPV was the most challenging vein, with the highest reconnection rate compared to other veins in both groups and a significantly lower reconnection rate in the nMARQ group compared to the TC group (18\% versus $28 \%, \mathrm{p}=0.05)$, respectively, as shown in figure 8.

\section{Radiofrequency Duration in PV}

$\mathrm{RF}$ duration at repeat procedure was not significantly different between groups as shown in table 4 in the supplementary file. RPV RF duration was $8 \pm 5$ minutes; LPV RF duration was $7 \pm 8$ minutes in nMARQ and $10 \pm 6,10 \pm 7$ minutes in the TC group.

\section{Repeat Procedure Parameters}

Total procedure time was significantly lower in patients initially treated with nMARQ $(142 \pm 56$ min versus $186 \pm 76, p=$ $0.001)$, respectively. Total RF (16 \pm 13 versus $23 \pm 16, p=0.072)$ and X-ray exposure ( $35 \pm 25$ min versus $48 \pm 32, p=0.084$ ), were shorter in nMARQ versus TC respectively, but not significantly shown in table 5 in the supplementary file.

There was no link between PV reconnection per vein or per segment between index and repeated procedures (Cohen's kappa $=0.048$ and -0.042 ).

\section{Clinical Follow Up}

The success rate at 1 year was $77 \%$ of patients in nMARQ group and $79 \%$ in TC group. AF recurrence (symptomatic and by Holter monitor) at 3, 6 and 12 months are shown in table 6 in the supplementary file. At 12 months, data was available for both groups in 73 patients in whom 15 patients (21\%) had still AF. Eight patients had PAF and 7 PsAF (2 of these patients had valvular heart disease).

\section{Safety Outcomes, Adverse Events and Complications}

Major adverse events occurred in 2 patients (3\%), LIPV stenosis with LSPV thrombosis 2 months after redo procedure in a patient from the TC group, and renal infarction due to embolism 8 months after repeat ablation due to AF recurrence in nMARQ group. The patient who had LIPV stenosis with LSPV thrombosis (1 year after redo ablation) received a LIPV angioplasty with Bare Metal Stent (BMS) Cook 8 mm X 20 mm.

Two patients (3\%) had puncture-related complications after repeat procedures, including 1 arteriovenous fistula and 1 femoral artery aneurysm. One (1\%) patient had an acute pericardial effusion in nMARQ group during the index procedure. He was percutaneously drained with no consequences. There were no incidences of stroke, phrenic nerve paralysis or esophageal fistula.

\section{Discussion}

\section{Major Findings of this Study}

1. This randomized study demonstrates that the nMARQ catheter is 3 times faster in isolating PVs, while preserving efficacy. This circular ablation catheter demonstrated shorter RF time in all veins, shorter procedure duration and less x-ray exposure, compared to conventional catheter in the index procedure.

2. In index procedures, additional ablation at non-PV sites (when needed) was faster when ablated with nMARQ catheter. 
Table 4: Follow-up

\begin{tabular}{|c|c|c|c|}
\hline 3 months n (\%) & nMARQ (n 37) & Navistar TC (n 43) & p \\
\hline Symptoms & $17(46)$ & $16(37)$ & 0.429 \\
\hline Holter + & $18(49)$ & $15(35)$ & 0.212 \\
\hline 6 months & nMARQ (n 32) & Navistar TC (n 39) & 0.432 \\
\hline Symptoms & $5(16)$ & $9(23)$ & 0.978 \\
\hline Holter + & $5(16)$ & Navistar TC (n 38) & $\mathbf{p}$ \\
\hline 12 months & nMARQ (n 35) & $12(32)$ & 0.78 \\
\hline Symptoms & $10(29)$ & $8(21)$ & 0.852 \\
\hline
\end{tabular}

Supplementary Table 4: RF duration in repeated procedure

\begin{tabular}{|c|c|c|c|}
\hline min \pm SD & nMARQ (n 25) & Navistar TC (n 28) & p \\
\hline RSPV & $7 \pm 5$ & $6 \pm 5$ & 0.744 \\
\hline RIPV & $7 \pm 5$ & $6 \pm 5$ & 0.843 \\
\hline LSPV & $7 \pm 7$ & $9 \pm 5$ & 0.619 \\
\hline LIPV & $5 \pm 6$ & $5 \pm 4$ & 0.791 \\
\hline RPV & $8 \pm 5$ & $10 \pm 6$ & 0.239 \\
\hline LPV & $7 \pm 8$ & $10 \pm 7$ & 0.308 \\
\hline
\end{tabular}

Supplementary Table 5: Reconnection rate per PV segment in repeated procedure*

\begin{tabular}{|c|c|c|c|c|}
\hline \multicolumn{2}{|c|}{ n (\%) } & \multirow{2}{*}{$\begin{array}{c}\text { nMARQ (n 30) } \\
5(17)\end{array}$} & \multirow{2}{*}{$\begin{array}{c}\text { Navistar TC (n 32) } \\
5(17)\end{array}$} & \multirow{2}{*}{$\begin{array}{l}\mathbf{p} \\
1\end{array}$} \\
\hline \multirow{4}{*}{ RSPV } & Superior & & & \\
\hline & Anterior & $3(10)$ & $6(20)$ & 0.279 \\
\hline & Inferior & $5(17)$ & $3(10)$ & 0.448 \\
\hline & Posterior & $4(13)$ & $9(30)$ & 0.117 \\
\hline \multirow{4}{*}{ RIPV } & Superior & $3(10)$ & $3(9)$ & 0.933 \\
\hline & Anterior & $6(20)$ & $9(28)$ & 0.455 \\
\hline & Inferior & $8(27)$ & $13(41)$ & 0.246 \\
\hline & Posterior & $4(13)$ & $11(34)$ & 0.053 \\
\hline \multirow{4}{*}{ LSPV } & Superior & $7(23)$ & $6(19)$ & 0.658 \\
\hline & Anterior & $3(10)$ & $13(41)$ & 0.006 \\
\hline & Inferior & $3(10)$ & $2(6)$ & 0.588 \\
\hline & Posterior & $6(20)$ & $6(19)$ & 0.901 \\
\hline \multirow{4}{*}{ LIPV } & Superior & $4(13)$ & $3(9)$ & 0.622 \\
\hline & Anterior & $2(7)$ & $3(9)$ & 0.696 \\
\hline & Inferior & $5(17)$ & $4(13)$ & 0.642 \\
\hline & Posterior & $4(13)$ & $5(16)$ & 0.798 \\
\hline
\end{tabular}

Per PV segment, RIPV posterior segment and LSPV anterior segment showed lower reconnection rate in nMARQ compared to TC (13\% versus $34 \%$, $\mathrm{p}=0.053$ and $10 \%$ versus $41 \% \mathrm{p}=0.006$ ), respectively. Among all veins segments for each group, the RIPV inferior segment in both groups and the LSPV anterior segment in TC group only had higher reconnection rates 


\begin{tabular}{|c|c|c|c|}
\hline \multicolumn{4}{|c|}{ Supplementary Table 6: Number of PV segment reconnection rate per patient in repeated procedure* } \\
\hline $\mathrm{n} / 16(\%)$ & nMARQ (n 30) & Navistar TC (n 32) & $\mathbf{p}$ \\
\hline $\mathbf{0}$ & $5(17)$ & $4(13)$ & 0.728 \\
\hline 1 & $5(17)$ & $3(9)$ & 0.467 \\
\hline 2 & $8(27)$ & $8(25)$ & 1 \\
\hline 3 & $5(17)$ & $7(22)$ & 0.751 \\
\hline 4 & $4(13)$ & $3(9)$ & 0.703 \\
\hline 5 & - & $1(3)$ & 1 \\
\hline 6 & $1(3)$ & $2(6)$ & 1 \\
\hline 7 & $2(7)$ & $1(3)$ & 0.607 \\
\hline 8 & - & $2(6)$ & 0.492 \\
\hline 9 & - & $1(3)$ & 1 \\
\hline $\begin{array}{l}\text { Total PV segment } \\
\text { reconnection }\end{array}$ & $25(83)$ & 27 (87) & 0.728 \\
\hline $\begin{array}{c}\text { Total PV segment } \\
\text { reconnection extent, } n \pm S D\end{array}$ & $2 \pm 2$ & $3 \pm 2$ & 0.177 \\
\hline \multicolumn{4}{|c|}{$\begin{array}{l}\text { Per patient, no statistically significant difference was observed between groups. Zero reconnection was found in } 17 \% \text { versus } 13 \% \text { of patients in } \\
\text { nMARQ and TC groups, respectively. The majority of patients in both groups had reconnection in two segments per vein. Nine } / 16 \text { reconnected PV } \\
\text { segments was the maximum number of reconnections. Total PV reconnection extent was } 2 \pm 2 \text { segments versus } 3 \pm 2 \text { segments in the nMARQ and } \\
\text { TC groups, respectively ( } \mathrm{p}=0.177 \text { ) }\end{array}$} \\
\hline
\end{tabular}

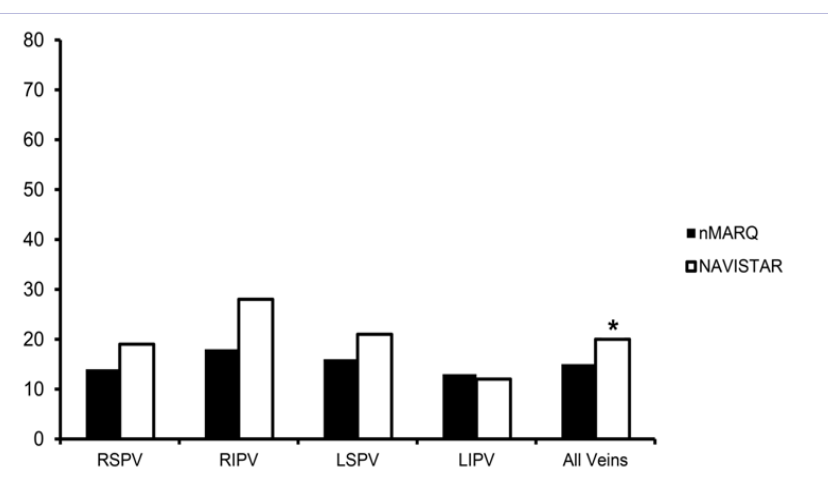

Figure 8: Percentage of reconnected pulmonary vein segments in repeated procedure

3. Our study benefited from a high acceptance rate for systematic $\mathrm{PV}$ assessment at 3 months. It allowed to demonstrate fewer PV reconnections at 3 months in the nMARQ group.

4. RIPV reconnection was less common after ablation with the nMARQ catheter, both acutely and at 3 months.

5. Patients ablated with the circular catheter also had shorter repeat procedures suggesting more discrete PV reconnections.

6. The circular ablation catheter appeared to be safe with no major procedural complications.

\section{PV Reconnections, Acutely and At 3 Months}

Our results are in line with several studies which have demonstrated that PVI using nMARQ is feasible and acutely successful in 96 to $100 \%$ [10-14].
The RF duration to isolate the veins has been reported to vary from 10 to 14 minutes, with procedure time from 86 to 131 minutes and $\mathrm{X}$ ray exposure from 4 to 26 minutes $[10-12,15,16]$.

Acute reconnection has been reported in $22 \%$ (Lasso only) to $42 \%$ when adenosine was used $[10,12]$. Our findings confirm that, when using the nMARQ catheter, the RSPV is the most challenging vein, requiring more $\mathrm{RF}$ applications and with a higher acute reconnection rate per vein and per segment; particularly at the anterior segment. On the other hand, RIPV showed a significantly lower reconnection rate per vein in nMARQ acutely and at 3 months, particularly at the posterior segment when compared to TC.

Our study shows heterogeneous efficacy of the nMARQ catheter, with superiority on the RIPV, particularly on the posterior segment, and at the ridge between LAA and LSPV. This may reflect better catheter stability and contact. This observation may guide improvements in performance on other veins and segments.

In a large, multicentre study, 374 patients underwent PVI with nMARQ for PAF and persistent AF (PsAF) in 5 centers with an acute PVI rate of $99.6 \%$. Again, RF times were longer in the superior veins compared to the inferior veins (LSPV $4 \pm 3$, RSPV $4 \pm 2$, LIPV and RIPV $3 \pm 2$ minutes, $p=0.0001$ ). And the anterior aspect of RSPV lesion was also identified as a weak point using this catheter.

$\mathrm{PV}$ reconduction is the dominant finding at repeat procedures for $\mathrm{AF}$ recurrence, and has been observed in $94 \%$ of patients after point-by-point ablation; which may reflect areas of poor catheter contact and lesion discontinuity [3]. 
Different "one shot" catheter designs and technologies aiming at reducing procedure and fluoroscopy time for PVI have been considered and tested over the last decade. In our study, procedure time to achieve PVI with nMARQ was $111 \pm 35$ minutes, with fluoroscopy of $26 \pm 11$ minutes. In a meta-analysis of 23 cryoablation studies, the mean procedure time was $206 \pm$ 72 minutes, with fluoroscopy of $46 \pm 13$ minutes [17]. In another meta-analysis of 42 studies of AF ablation with PVAC catheter average procedure time were reported as $117 \pm 33$ minutes, with fluoroscopy of $27 \pm 10$ minutes [18].

\section{Safety and Complications}

Several recent studies have reported on outcomes and complication rates following ablation with the nMARQ catheter $[10-14,16,19,20]$. Of 894 patients included in 8 studies, major complications were observed in 6 patients $(0.7 \%)$ including 4 deaths following nMARQ ablation. Three patients died from oesophago-pericardial fistula and 1 had fatal sepsis from an unknown source $[11,16]$. The mortality rate with the nMARQ catheter seems higher than the $0.1 \%$ reported with conventional catheters [21]. However, it is important to highlight that the latter involved a larger population ( $>30,000$ patients) and was a registry based on physician willingness to declare complications. Oesophageal lesions detected by endoscopy were attributed to the use of higher RF power in the posterior wall and prolonged RF duration delivery $[13,16]$. In our study, no major complications or mortalities were identified with nMARQ catheter except for one acute cardiac tamponade during the initial procedure $1 / 38$. However, pulmonary vein stenosis (TC group) and renal embolism (nMARQ group) due to AF recurrence occurred after redo procedures with the conventional catheter. However, we didn't use oesophageal temperature monitoring or systematically perform post ablation endoscopic evaluation to document thermal oesophageal damages. Studies in larger cohorts of patients are necessary to define the mortality rate associated with nMARQ ablation.

Serious safety concerns regarding Silent Cerebral Lesions (SCL) caused by multi-electrode ablation in the LA have arisen recently $[22,23]$. Scaglione reported no major procedural complications and no SCL by 24 hours post procedural cerebral diffusion-weighted DW-MRI, in contrast with the results reported by Deneke which showed that 14 out of 43 patients (33\%) had SCL following nMARQ ablation $[12,13]$. We did not routinely acquire cerebral imaging post ablation.

The complication rate in cryoablation reported in a systematic review was relatively low with $6 \%$ incidence of Phrenic Nerve Palsy (PNP), most of them being transient [17]. In a systematic review of PVAC ablation, the overall procedural complication rate appeared to be low (acute procedural complications were $2 \%$, though non-uniform screening may limit the accuracy of this estimate). However, high rates of silent cerebral ischemic lesions (38\%-45\%) have been reported, significantly higher than with irrigated RF (7\%-17\%) or cryoablation (4\%-6\%) [18].

\section{Long-Term Outcome}

A single procedure 1-year success rate of $65 \%$ has been reported using nMARQ, similar to the $67 \%$ reported in a meta- analysis on conventional RF catheters $[11,24]$. Success rate after single and repeat procedures was 66 and $77 \%$ respectively, similar to the success rates reported in conventional RF and multi-electrode ablation and higher than in cryoablation [25,26].

Another single operator study reported $83 \%$ success rate at 1 year with the nMARQ [16]. In our study, $77 \%$ of patients in nMARQ group and $79 \%$ in TC group were in SR $(p=0.852)$ at 1 year. Long-term success $(\approx 18$ months) with freedom from $\mathrm{AF}$, AFL or AT was $80 \%$ in a study comparing nMARQ to TC Smart Touch in 86 patients with PAF and PsAF with no significant difference in both groups $(p=0.78)$ [15].

\section{Limitations}

Our study is a single centre randomized study that did not meet the planned enrolment target. The catheter withdrawal from the market in June 2015 interrupted patient recruitment to the study. However, the patient cohort recruited has been sufficiently large to demonstrate statistically significant results.

We did not routinely monitor oesophageal temperature during ablation or perform post ablation esophageal endoscopy or cerebral imaging (MRI). However, we did not observe any oesophageal or cerebral complications, possibly as a consequence of reduced power and duration of RF delivery in the posterior LA.

Another potential limitation was in the technology used in conventional single electrode catheters. We used an impedancebased method rather than direct measurements of force sensing technologies 'contact force' that associated with a more complete ablation.

Our observation of nMARQ efficacy and safety suggests that this catheter may be a promising alternative to conventional point-by-point $\mathrm{AF}$ ablation and the knowledge gained using this catheter should be used to build better ablation tools in the future.

\section{Conclusion}

This randomized study shows that the nMARQ circular irrigated catheter is 3 times faster in isolating the PVs, with shorter RF and procedure time. PVI using a circular ablation catheter is also associated with fewer PV reconnections at 3 months. This study may provide guidance for future circular ablation catheter designs as demonstrated by superior efficacy in RIPV and suboptimal results in RSPV.

\section{Conflict of Interest}

Dr. Nora Al-Jefairi has no conflict of interest. Drs. Pierre Jais, Frederic Sacher, Meleze Hocini and Michel Haissaguerre have received lecture fees from Biosense Webster for less than 10.000 USD annually. Other authors have no conflict of interest to disclose concerning this paper.

\section{Funding}

The research leading to these results has been partially funded by the European Union Seventh Framework Programme (FP7/2007-2013) under Grant Agreement HEALTH-F2-2010-261057. 


\section{References}

1. Fuster V, Ryden LE, Asinger RW, Cannom DS, Crijns HJ, Frye RL, et al. ACC/AHA/ESC Guidelines for the Management of Patients With Atrial Fibrillation: Executive Summary A Report of the American College of Cardiology/American Heart Association Task Force on Practice Guidelines and the European Society of Cardiology Committee for Practice Guidelines and Policy Conferences (Committee to Develop Guidelines for the Management of Patients With Atrial Fibrillation) Developed in Collaboration With the North American Society of Pacing and Electrophysiology. Circulation. 2001. 104:2118-2150.

2. Wilber DJ, Pappone C, Neuzil P, De Paola A, Marchlinski F, Natale A, et al. Comparison of antiarrhythmic drug therapy and radiofrequency catheter ablation in patients with paroxysmal atrial fibrillation: a randomized controlled trial. JAMA. 2010;303(4):333-340. doi: 10.1001/jama.2009.2029

3. Ouyang F, Tilz R, Chun J, Schmidt B, Wissner E, Zerm T, et al. Long-term results of catheter ablation in paroxysmal atrial fibrillation: lessons from a 5-year follow-up. Circulation. 2010;122(23):2368-2377. doi: 10.1161/CIRCULATIONAHA.110.946806

4. Hussein AA, Saliba WI, Martin DO, Bhargava M, Sherman M, MagnelliReyes C, et al. Natural history and long-term outcomes of ablated atrial fibrillation. Circ Arrhythm Electrophysiol. 2011;4(3):271-278. doi: 10.1161/CIRCEP.111.962100

5. Natale A, Raviele A, Arentz T, Calkins H, Chen SA, Haissaguerre M, et al. Venice Chart international consensus document on atrial fibrillation ablation. J Cardiovasc Electrophysiol. 2007;18(5):560-580.

6. Daubert JC, Saxon L, Adamson PB, Auricchio A, Berger RD, Beshai JF, et al. 2012 EHRA/HRS expert consensus statement on cardiac resynchronization therapy in heart failure: implant and follow-up recommendations and management. Europace. 2012;14(9):12361286. doi: 10.1093/europace/eus222

7. Calkins H, Kuck KH, Cappato R, Brugada J, Camm AJ, Chen SA, et al. 2012 HRS/EHRA/ECAS expert consensus statement on catheter and surgical ablation of atrial fibrillation: recommendations for patient selection, procedural techniques, patient management and follow-up, definitions, endpoints, and research trial design: a report of the Heart Rhythm Society (HRS) Task Force on Catheter and Surgical Ablation of Atrial Fibrillation. Developed in partnership with the European Heart Rhythm Association (EHRA), a registered branch of the European Society of Cardiology (ESC) and the European Cardiac Arrhythmia Society (ECAS); and in collaboration with the American College of Cardiology (ACC), American Heart Association (AHA), the Asia Pacific Heart Rhythm Society (APHRS), and the Society of Thoracic Surgeons (STS). Endorsed by the governing bodies of the American College of Cardiology Foundation, the American Heart Association, the European Cardiac Arrhythmia Society, the European Heart Rhythm Association, the Society of Thoracic Surgeons, the Asia Pacific Heart Rhythm Society, and the Heart Rhythm Society. Heart Rhythm. 2012;9(4):632696.e21. doi: 10.1016/j.hrthm.2011.12.016

8. Weerasooriya R, Khairy P, Litalien J, Macle L, Hocini M, Sacher F, et al. Catheter ablation for atrial fibrillation: are results maintained at 5 years of follow-up? J Am Coll Cardiol. 2011;57(2):160-166. doi: 10.1016/j.jacc.2010.05.061
9. Wong CX, Abed HS, Molaee P, Nelson AJ, Brooks AG, Sharma G, et al. Pericardial fat is associated with atrial fibrillation severity and ablation outcome. J Am Coll Cardiol. 2011;57(17):1745-51. doi: 10.1016/j.jacc.2010.11.045

10.Zellerhoff S, Daly M, Lim HS, Denis A, Komatsu Y, Jesel L, et al. Pulmonary vein isolation using a circular, open irrigated mapping and ablation catheter (nMARQ): a report on feasibility and efficacy. Europace. 2014;16(9):1296-1303. doi: 10.1093/europace/euu133

11. Mahida S, Hooks DA, Nentwich K, Ng GA, Grimaldi M, Shin DI, et al. nMARQ Ablation for Atrial Fibrillation: Results from a Multicenter Study. J Cardiovasc Electrophysiol. 2015;26(7):724-729. doi: 10.1111/ jce. 12698

12. Scaglione M, Caponi D, Anselmino M, Di Clemente F, Blandino A, Ferraris $\mathrm{F}$, et al. Pulmonary vein isolation with a new multipolar irrigated radiofrequency ablation catheter (nMARQ): feasibility, acute and short-term efficacy, safety, and impact on postablation silent cerebral ischemia. J Cardiovasc Electrophysiol. 2014;25(12):12991305. doi: $10.1111 /$ jce. 12500

13. Deneke T, Schade A, Muller P, Schmitt R, Christopoulos G, Krug J, et al. Acute safety and efficacy of a novel multipolar irrigated radiofrequency ablation catheter for pulmonary vein isolation. J Cardiovasc Electrophysiol. 2014 Apr;25(4):339-45. doi: 10.1111/ jce.12316

14. Rosso R, Chorin E, Levi Y, Rogowski O, Viskin S. Radiofrequency Ablation of Atrial Fibrillation: Nonrandomized Comparison of Circular versus Point-by-Point "Smart” Ablation for Achieving Circumferential Pulmonary Vein Isolation and Curing Arrhythmic Symptoms. J Cardiovasc Electrophysiol. 2016. doi: 10.1111/jce.13058

15. Rosso R, Halkin A, Michowitz Y, Belhassen B, Glick A, Viskin S. Radiofrequency ablation of paroxysmal atrial fibrillation with the new irrigated multipolar nMARQ ablation catheter: Verification of intracardiac signals with a second circular mapping catheter. Heart Rhythm. 2014;11(4):559-565. doi: 10.1016/j.hrthm.2013.12.029

16. Martin Vurma, Lam Dang, Hans-Peter Brunner-La Rocca, Gabor Sutsch, Christine H Attenhofer-Jost, Firat Duru, et al. Safety and efficacy of the nMARQ catheter for paroxysmal and persistent atrial fibrillation. Europace. 2016;18(8):1164-1169.

17. Andrade JG, Khairy P, Guerra PG, Deyell MW, Rivard L, Macle L, et al., Efficacy and safety of cryoballoon ablation for atrial fibrillation: a systematic review of published studies. Heart Rhythm. 2011;8(9):1444-1451. doi: 10.1016/j.hrthm.2011.03.050

18. Andrade JG, Dubuc M, Rivard L, Guerra PG, Mondesert B, Macle L, et al. Efficacy and safety of atrial fibrillation ablation with phased radiofrequency energy and multielectrode catheters. Heart Rhythm. 2012;9(2):289-296. doi: 10.1016/j.hrthm.2011.09.009

19. Shin DI, Kirmanoglou K, Eickholt C, Schmidt J, Clasen L, Butzbach B, et al. Initial results of using a novel irrigated multielectrode mapping and ablation catheter for pulmonary vein isolation. Heart Rhythm. 2014;11(3):375-383. doi: 10.1016/j.hrthm.2013.12.008

20. Rillig A, Lin T, Burchard A, Kamioka M, Heeger C, Makimoto H, et al. Modified energy settings are mandatory to minimize oesophageal injury using the novel multipolar irrigated radiofrequency ablation 
catheter for pulmonary vein isolation. Europace. 2015;17(3):396-402. doi: 10.1093/europace/euu269

21. Cappato R, Calkins H, Chen SA, Davies W, Iesaka Y, Kalman J, et al. Prevalence and causes of fatal outcome in catheter ablation of atrial fibrillation. J Am Coll Cardiol. 2009;53(19):1798-1803. doi: 10.1016/j. jacc.2009.02.022

22. Herrera Siklody C, Deneke T, Hocini M, Lehrmann H, Shin DI, Miyazaki $\mathrm{S}$, et al. Incidence of asymptomatic intracranial embolic events after pulmonary vein isolation: comparison of different atrial fibrillation ablation technologies in a multicenter study. J Am Coll Cardiol. 2011;58(7):681-688. doi: 10.1016/j.jacc.2011.04.010

23. Stephan Zellerhoff, Martin A Ritter, Simon Kochhauser, Ralf Dittrich, Julia Kobe, Peter Milberg, et al. Modified phased radiofrequency ablation of atrial fibrillation reduces the number of cerebral microembolic signals. Europace. 2014;16(3):341-346.
24. Ganesan AN, Shipp NJ, Brooks AG, Kuklik P, Lau DH, Lim HS, et al. Longterm outcomes of catheter ablation of atrial fibrillation: A systematic review and meta analysis. J Am Heart Assoc. 2013;2(2):e004549. doi: 10.1161/JAHA.112.004549

25. Packer DL, Kowal RC, Wheelan KR, Irwin JM, Champagne J, Guerra PG, et al. Cryoballoon ablation of pulmonary veins for paroxysmal atrial fibrillation: First results of the North American Arctic Front (STOP AF) pivotal trial. J Am Coll Cardiol. 2013;61(16):1713-1723. doi: 10.1016/j.jacc.2012.11.064

26. Bittner A, Monnig G, Zellerhoff S, Pott C, Kobe J, Dechering D, et al. Randomized study comparing duty-cycled bipolar and unipolar radiofrequency with point-by-point ablation in pulmonary vein isolation. Heart Rhythm. 2011;8(9):1383-1390. doi: 10.1016/j. hrthm.2011.03.051 